\title{
Toxicology Case Files: Case Records from Medical Toxicology Fellowship Programs
}

Beginning with the next issue of the Journal, the Toxicology Case Files will be a regular feature within the Journal of Medical Toxicology, and will highlight concepts and controversies in medical toxicology culled from real cases. Each issue will feature a different case presentation from a medical toxicology fellowship training program. Dr. Jeffrey Suchard, MD, FACMT, will be the first Feature Editor for the Toxicology Case Files, providing the Feature with organization and oversight. The Journal hopes that this Feature will stimulate the reader's interest, and provide an academic experience to some of our Medical Toxicology Fellowsin-Training. 